Review Article

\section{Current status, complications and prospects of fecal microbiota transplantation therapy}

\author{
Tadashi Ohara*
}

Department of Gastroenterology, Kitashinagawa Third Hospital, Kohno Clinical Research Institute, Japan

\section{Abstract}

Currently, the emergence of highly virulent mutants in Europe and the United States has caused refractory recurrent Clostridium difficile infection (RCDI) to be a problem in clinical practice. In 2013, the Netherland group demonstrated breakthrough therapeutic efficacy in fecal microbial transplant (FMT) treatment clinical trials for RCDI, and FMT treatment is rapidly gaining attention. In addition to RCDI, FMT treatment has been attempted in various gastrointestinal diseases such as inflammatory bowel disease, irritable bowel syndrome and chronic constipation, as well as extragastrointestinal diseases. In this review, I would like to describe the current status, complications and prospects of FMT treatment.

\section{Introduction}

Fecal Microbiota Transplantation (FMT) treatment is attracting attention. In 2013, a team from the Amsterdam Medical Center in the Netherlands randomized comparisons of refractory recurrent Clostridium difficile infection (RCDI) in 2013. In a randomized controlled trial (RCT), breakthrough results were reported in The New England Journal of Medicine [1]. This work was a breakthrough in terms of clinical application of enterobacterial research [2], which had emerged due to the spread of next-generation sequencing technology and intestinal bacterial analysis technology using sterile mice. In recent years, FMT is being tried for various diseases other than RCDI [3]. However, there are many unsolved problems, such as the mechanism of symbiotic ecosystems between enteric bacteria and host $[4,5]$, and the whole picture of the metabolites produced by enteric bacteria and the interaction between bacteriophages and fungi [6,7]. In this review, I will explain the history and current status of FMT treatment for many medical staff, including physicians in the field of gastroenterology, and consider the clinical application of FMT treatment.

\section{History of FMT treatment}

FMT treatment has an old history in human society, and there is a document that it was already performed in China in the 4th century AD. A scholar named Ge Hong in a book on Chinese emergency medicine administers feces of healthy

\section{More Information}

*Address for Correspondence: Tadashi Ohara M.D., Ph.D. Department of Gastroenterology, Kitashinagawa Third Hospital, Kohno Clinical Research Institute, 3-3-7 Kitashinagawa, Shinagawa-Ku, Tokyo 140-0001, Japan, Tel: +81-3-3474-1831; Fax: +81-3-5461-3740; Email: t.ohara@kcmi.or.jp

Submitted: April 02, 2021 Approved: April 14, 2021 Published: April 15, 2021

How to cite this article: Ohara T. Current status, complications and prospects of fecal microbiota transplantation therapy. Arch Pathol Clin Res. 2021; 5: 004-009.

DOI: 10.29328/journal.apcr.1001021

ORCiD: orcid.org/0000-0002-7039-8144

Copyright: (c) 2021 Ohara T. This is an open access article distributed under the Creative Commons Attribution License, which permits unrestricted use, distribution, and reproduction in any medium, provided the original work is properly cited.

Keywords: Fecal microbiota transplantation treatment; Ulcerative colitis; Crohn's disease; Irritable bowel syndrome; Chronic constipation; Intestinal flora; Clostridium difficile infection; Dysbiosis

D) Check for updates

(1) Open Access

individuals to food poisoning or acute diarrhea [8]. There is also a custom among the Middle Eastern nomads, Bedouin, to consume fresh camel feces when bacillary dysentery is present. The outbreak of bacterial dysentery among German soldiers stationed in Africa during World War II caused all soldiers who had consumed fresh camel feces to survive, and all soldiers who had not consumed them to have died. They proved the effects of their customs. For the first time in the literature, a surgeon named Eiseman performed FMT therapy on four patients with pseudomembranous enteritis in 1958 [9]. However, FMT therapy has disappeared from the main stage in human society and has only been performed sporadically. The reason for this event may be that various antibiotics have been treated to microbial infections, starting with the discovery of penicillin.

\section{Current status of FMT treatment}

FMT treatment in RCDI: Van Nood, et al.'s report of an RCT study on RCDI marked a breakthrough in FMT treatment [1]. The background of this study was largely related to the emergence of NAP1/027 mutants that produce high toxins 
(toxin A/B and binary toxin) in Europe and the United States. Mutant strain NAP1/027 is resistant to conventional antibiotics such as metronidazole and vancomycin and is prone to recurrence, each time becoming more severe and intractable, and often fatal. For this reason, the mutant strain NAP1/027 is a bacterium that has become of tremendous medical and social interest [10]. The use of antibiotics as a risk factor for CDI is well known, but postoperative, perinatal, occupation in nursing homes, inflammatory bowel disease (IBD), and oral use of PPIs require attention and attention [11]. These risk factors can induce stress on the body and disruption of the intestinal mucosal barrier and have a negative effect on the intestinal flora. Indeed, it is well known that CDI patients have dysbiosis that is far from normal intestinal flora, and that the diversity of intestinal flora is extremely reduced. The German research team mentioned earlier showed that the 4-day antibiotics plus FMT treatment group (81\% recurrence-free rate) suppressed relapse with an overwhelmingly significant difference compared to the 14-day antibiotic-only group (31\% recurrence-free rate) [1].

By the way, what is the treatment of donor fecal microorganisms in healthy people equivalent from the medical point of view? Drugs? Natural product medicine? Food? material? The U.S. Food and Drug Administration (FDA) issued a guideline regarding the use of drugs that require an IND (investigative new drug) procedure [12]. This problem has not been solved yet. The FDA is now accepting FDI treatment for RCDI after rigorous explanation and informed consent (IC) with the approval of an ethical review in a healthcare facility. As a result, a number of RCTs have replicated the proof of RCDI effectiveness, and metaanalysis results have demonstrated its effectiveness [13]. FMT treatment for RCDI is now the standard treatment in Europe and the United States. In addition, various guidelines have been published in Europe and the United States [14]. Even in the first case, it is recommended that FMT therapy be continued if the patient is resistant to antibiotic therapy [4]. However, it is considered that there is no problem with treatment skipping to FMT treatment after resistance has been found by antibiotic treatment.

I would like to emphasize again that FMT treatment is not allowed. In Japan, the establishment of a treatment system is delayed, and urgent action is desired. In Japan, as in Europe and the United States, RCDI is currently subject to rigorous IC approval with the approval of ethical review.

In the United States and Europe, attempts are being made to encapsulate feces [15]. By taking cryopreserved fecal capsules, good results equivalent to the administration of fresh stool have been obtained by RCDI [16]. Although fecal capsules may support the spread of FMT treatment, the problem of whether processed capsules are pharmaceuticals has not been solved. It is also expected that the situation will change depending on whether companies are developing capsules as a business or nonprofit organization (NPO). The current status of FMT treatment in Japan has not been established, including the risk management of transmission of infectious diseases and other diseases, and it is hoped that clinical trials will be conducted under strict rules. Unresolved issues remain in the protocol for FMT treatment of RCDI [17]. At the end of this section, we status the current thinking on below issues.

\section{a) Feces of donor used for FMT treatment}

RCDI has demonstrated that both fresh and frozen stools are effective. Therefore, it is said that cryopreservation (feces bank), which performs sufficient infectious disease screening in advance, is excellent in safety, efficiency, and economy. In terms of efficiency, it is said that once frozen, the relative proportion of spore bacilli can be selectively increased, which may have an effect. Frozen flights are being offered to medical institutions across the United States for $\$ 250$ by an NPO corporation called Open Biome in Boston.

b) Selection of feces as a donor

At the beginning of FMT treatment, feces of relatives within the second degree of the patient were selected as donors. However, after finding out that cryopreserved feces are superior in safety, efficiency and economy, facilities using third party feces are gradually increasing. Intestinal flora in faces of relatives who are expected to live in a similar lifestyle may be similar to those of patients, but there are many negative reports at this time. On the other hand, there is a report [18] that the close genetic background enhances the homology of the intestinal flora. In this regard, the use of third-party feces rather than relatives' feces increases diversity and get easily.

\section{c) Transplant route of FMT treatment}

There have been many reports in the RCDI that transanal administration (enema or lower gastrointestinal endoscopy) is more effective than oral administration using a duodenal tube [19]. The most effective treatment is in the ileocecal region using a lower gastrointestinal endoscope, but if the risk of perforation is suspected, enema is recommended.

\section{d) Numbers of FMT treatment}

It is often cured with a single FMT treatment. If healing is difficult at one time, a second FMT treatment using the same donor's feces are performed, but if treatment is also difficult with the second FMT treatment, the option of using feces from other donors are also recommended.

\section{e) Administration of antibiotics before FMT treatment}

For both recurrent and intractable CDI, antibiotics are recommended for 3 to 5 days. It may be given to confirm antibiotic resistance and to reduce patient-specific intestinal 
bacterial counts. It is also recommended to treat FMT 24 to 36 hours after the last dose so that donor bacteria will not be killed by antibiotics.

FMT treatment for gastrointestinal diseases other than RCDI: Due to the success of FMT treatment in RCDI, an attempt is being made to apply it to intractable diseases associated with dysbiosis and reduced diversity of intestinal flora, similar to RCDI [20]. Here, we introduce FMT treatment for ulcerative colitis (UC), Crohn's disease (CD), and irritable bowel syndrome (IBS), in which the presence of intestinal flora dysbiosis has been identified. Four RCTs (of which one is a conference report) have been reported in the FMT at UC [21,23], and meta-analysis has already been performed based on these RCTs, and it is considered to be effective [24]. However, unlike RCTs for pharmaceuticals, this is a small-scale meta-analysis without protocol unification, so it is important to accumulate data in the future, and do not rush to a conclusion. These studies suggest that UC should be treated with multiple, rather than single, FMT treatments at regular intervals, with early onset at mild to moderate stages, and selection of highly effective donors (search for a super donor) (21) may improve the effectiveness. However, I think that caution should be exercised in the treatment of FMT at the early stage of onset and in the mild to moderate stages. This is because there is too much safety concern in treating FMT by skipping existing proven drugs. It should be remembered that UC is a benign disease that mainly affects young people, whereas RCDI is a potentially fatal disease in Europe and the United States. According to Australian report, a total of 41 cocktails of donor's feces as FMT were given, with less than $30 \%$ steroid-free remission [25]. Although it is expected that there is maintenance of remission and mucosal healing after FMT treatment and the possibility of radical cure, it is not possible to judge it at this time. Only major adverse events such as exacerbation of abdominal disease and abdominal pain/diarrhea have been reported in this study, sufficient safety evaluation is necessary, including the possibility of transmission of infectious diseases and unknown diseases. Even though there is no report of RCT of FMT treatment including UC from Japan, several pilot studies in UC have been carried out, and the efficacy is reported to be approximately $10 \%$ to $30 \%[23,26]$. CD is also an intractable disease with dysbiosis and reduced diversity of intestinal flora. A meta-analysis that analyzed multiple pilot studies in 2014 showed that the effectiveness of CD was expected to be higher than that of UC [27], but no RCT has been reported so far, and only a small number of pilot studies are currently available [28]. In addition, the complexity of the affected area (small intestine, large intestine, small intestine and large intestine type) and the safety of FMT treatment due to the presence of fistulas and abscesses indicate the difficulty of RCT. On the other hand, the IBS reported a small but effective RCT in 2017 from the Norwegian group. This report found that the effective rate was $43 \%$ in the placebo group and $65 \%$ in the treatment group ( $p=0.049$ ), which was significantly higher [29]. Because IBS is also known for constipation, diarrhea, mixed, and complex placebo effects, it is necessary to wait for additional RCT results. In pilot studies, it is also recommended to perform graft-versus-host disease (GVHD), intestinal Bechet's disease, ileitis, and function.

All reports of FMT treatment for chronic constipation were effective and have no complications [30-32]. Regarding the number of FMT treatments, regular multiple treatments have been reported to be more effective than single treatment [31]. The author has reported the dramatic therapeutic effect of FMT treatment for a patient with chronic intractable constipation (Table 1) [33]. In addition, I have reported new findings that beneficial transplanted microorganisms related to intestinal peristalsis from a donor colonized the recipient's intestine for a long-term (Figure 1) [34]. Although above patient also had a dementia of Alzheimer's type, the patient's cognitive function improved after FMT treatment. These results also suggest that FMT treatment can be applied clinically to various diseases.

FMT treatmentfor diseases other than gastrointestinal tract: A brief overview of FMT in non-gastrointestinal disorders. In addition to RCDI and UC, RCT suggests efficacy in studies of patients with metabolic syndrome (obesity) [35,36] and hepatic encephalopathy [37]. The above RCT of RCDI was an open, unblinded study that was assigned randomization. On the other hand, it should be noted that the RCT for patients with metabolic syndrome was double-blind. In other words, the placebo group involves transplanting a patient's own feces sample collected in advance, which means that a protocol with a high level of evidence was used, as in the case of normal drug development. FMT for patients with metabolic syndrome did not improve obesity itself but did improve peripheral insulin sensitivity. In addition, although currently in pilot studies, drug-resistant hepatitis B, multiple sclerosis, dystonia, type 2 diabetes, acute GVHD, idiopathic thrombocytopenia, sepsis, multidrug-resistant bacterial infections, diseases such as nonalcoholic steatohepatitis (NASH), primary sclerosing cholangitis, acute pancreatitis,

\begin{tabular}{|l|l|l|}
\hline \multicolumn{1}{|c|}{ Item } & \multicolumn{1}{|c|}{ Before FMT } & After FMT1month \\
\hline $\begin{array}{l}\text { Abdominal } \\
\text { distension }\end{array}$ & Marked & None \\
\hline $\begin{array}{l}\text { Borborygmus } \\
\text { feeling }\end{array}$ & Almost nothing & Almost normal \\
\hline Use of laxative & Many laxatives & None \\
\hline Feces frequency & $0-1 /$ week & $1 /$ day \\
\hline Feces weight & Minimum & Moderate \\
\hline Feces odor & Offensive & Mild \\
\hline Feces color & Blackish-brown & Yellowish-brown \\
\hline Bristol Stool Scale & 1 & $3-4$ \\
\hline
\end{tabular}

Table 1: Changes of fecal properties and bowel movements in the recipient before and after FMT treatment. 


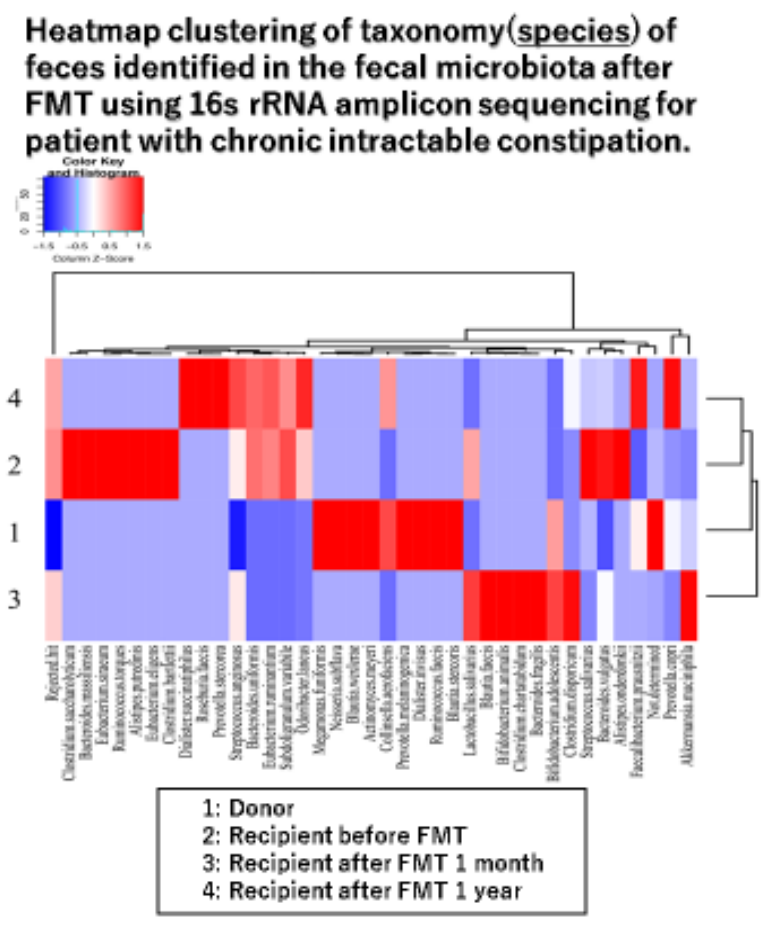

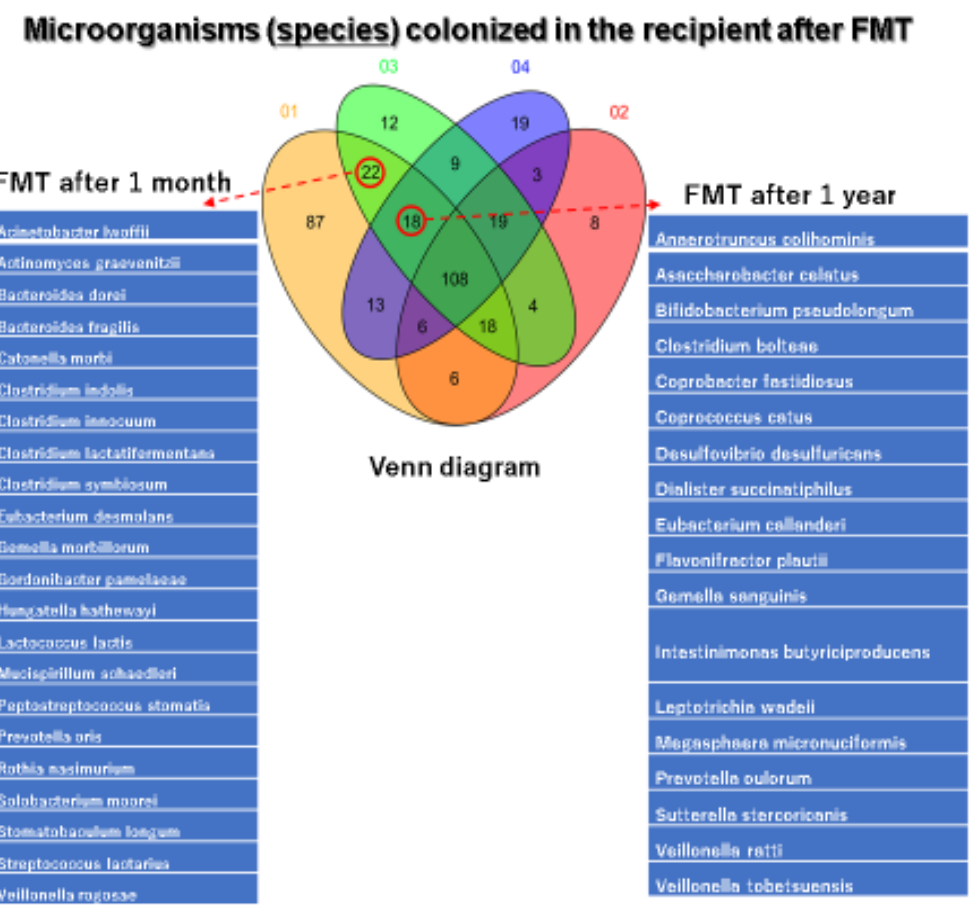

These microorganisms control genes involved in mucin degradation/biosynthesis (PICRUSt analysis).

Figure 1: The follow-up of transplanted microorganisms after FMT treatment.

Parkinson's disease, epilepsy, autism, depression, and HIV infection have been conducted in the context of clinical trials [38-41].

\section{Complications}

Recently, it has been reported that patients treated with FMT died of severe multidrug-resistant bacterial infections. It was that two immunodeficient patients treated with FMT from donors who had not tested for extended-spectrum $\beta$-lactamase (ESBL) -producing gram-negative bacteria developed an invasive infection with ESBL-producing Escherichia coli, one of whom died. The FDA has posted this case on its website and is calling attention [42]. Based on this case, the FDA will provide the FMT donor unless at least all of the ESBL-producing enterobacteria, vancomycin-resistant enterococci (VRE), carbapenem-resistant enterobacteria (CRE), and methicillin-resistant Staphylococcus aureus (MRSA) were negative. Notifying the study implementers of additional measures related to FMT clinical trials, such as the inability to obtain information and the need to explain the risk of severe multidrug-resistant bacterial infections to patients.

\section{Conclusion and prospects}

I described the current status and complications of FMT treatment and the future clinical application of FMT treatment. In Europe and the United States, the fierceness of RCDI has become a clinical problem. In the United States, the
NPO Open Biome Operates a fecal bank for RCDI [43]. Precise screening of infectious diseases in donor stool samples in advance and cryopreservation will greatly improve safety and emergency response capabilities. In Europe, the European Consensus Conference (EuCC) is the main organization, and the cooperation of each country is planned to operate a fecal bank [44]. It is easy to imagine that a large amount of funding is required for the operation of a fecal bank, but it is also desirable in each country to establish a system which can respond quickly of RCDI outbreaks. FMT treatment can be applied to many diseases such as gastrointestinal diseases as well as living diseases as introduced in this paper. Although specific cases of the complications of FNT treatment have been shown, it can be prevented by strictly checking infections when selecting feces of FMT donors. The most controversial and substantiated issues in FMT treatment from now on is whether the transplanted microorganisms of the donor will colonize the recipient's intestine over time. To date, only our report [34] has been a detailed study of the long-term colonization of transplanted microorganisms by FMT treatment. I would like to emphasize that this problem is an important issue which must be resolved in order to aim for clinical application of FMT treatment in various diseases in the future.

Finally, we look forward to the emergence of new therapy such as combine FMT treatment with biofeedback treatment which strengthens the pelvic floor muscles $[45,46]$. 


\section{References}

1. van Nood E, Vrieze A, Nieuwdorp M, Fuentes S, Zoetendal EG, et al. Duodenal infusion of donor feces for recurrent Clostridium difficile. $\mathrm{N}$ Engl J Med. 2013: 368, 407-415.

PubMed: https://pubmed.ncbi.nlm.nih.gov/23323867/

2. Martín R, Bermúdez-Humarán LG, Langella P. Gnotobiotic Rodents, An In Vivo Model for the Study of Microbe-Microbe Interactions. Front Microbiol. 2016; 7; 409.

PubMed: https://pubmed.ncbi.nlm.nih.gov/27065973/

3. Cammarota G, laniro G, Tilg H, Rajilić-Stojanović M, Kump P, et al. European consensus conference on faecal microbiota transplantation in clinical practice. Gut. 2017: 66, 569-580.

PubMed: https://pubmed.ncbi.nlm.nih.gov/28087657/

4. Rook G, Bäckhed F, Levin BR, McFall-Ngai MJ, McLean AR. Evolution, human-microbe interactions, and life history plasticity. Lancet. 2017; 390: 521-530.

PubMed: https://pubmed.ncbi.nlm.nih.gov/28792414/

5. Selber-Hnatiw S, Rukundo B, Ahmadi M, Akoubi $\mathrm{H}$, Al-Bizri $\mathrm{H}$, et al. Toward an Ecology of Disease. Front Microbiol. 2017; 1265.

6. Carding SR, Davis N, Hoyles L. Review article, the human intestinal viromeinhealthanddisease. AlimentPharmacolTher.2017;46:800-815. PubMed: https://pubmed.ncbi.nlm.nih.gov/28869283/

7. Mirzaei MK, Maurice CF. Ménage à trois in the human gut, interactions betweenhost, bacteriaand phages. NatRevMicrobiol.2017;15:397-408. PubMed: https://pubmed.ncbi.nIm.nih.gov/28461690/

8. Zhang F, Luo W, Shi Y, Fan Z, Ji G. Should we standardize the 1,700-year-old fecal microbiota transplantation? Am J Gastroenterol. 2012; 107: 1755.

PubMed: https://pubmed.ncbi.nlm.nih.gov/23160295/

9. Eiseman B, Silen W, Bascom GS, Kauvar AJ. Fecal enema as an adjunct in the treatment of pseudomembranous enterocolitis. Surgery. 1958; 44: 854-859.

PubMed: https://pubmed.ncbi.nlm.nih.gov/13592638/

10. O'Connor JR, Johnson S, Gerding DN. Clostridium difficile infection caused by the epidemic BI/NAP1/027 strain. Gastroenterology. 2009; 136: 1913-1924.

PubMed: https://pubmed.ncbi.nlm.nih.gov/19457419/

11. Tang YM, Stone $C D$. Clostridium difficile infection in inflammatory bowel disease, challenges in diagnosis and treatment. Clin $\mathrm{J}$ Gastroenterol. 2017; 10: 112-123.

PubMed: https://pubmed.ncbi.nlm.nih.gov/28210836/

12. Kelly CR, Kunde SS, Khoruts A. Guidance on preparing an investigational new drug application for fecal microbiota transplantation studies. Clin Gastroenterol Hepatol. 2014; 12: 283-288.

PubMed: https://pubmed.ncbi.nlm.nih.gov/24107393/

13. Quraishi MN, Widlak M, Bhala N, Moore D, Price M, et al. Systematic review with meta-analysis, the efficacy of faecal microbiota transplantation for the treatment of recurrent and refractory Clostridium difficile infection. Aliment Pharmacol Ther. 2017; 46: 479-493. PubMed: https://pubmed.ncbi.nlm.nih.gov/28707337/

14. Bakken JS, Borody T, Brandt LJ, Brill JV, Demarco DC, et al. Treating Clostridium difficile infection with fecal microbiota transplantation. Clin Gastroenterol Hepatol. 2011; 9: 1044-1049.

PubMed: https://pubmed.ncbi.nlm.nih.gov/21871249/

15. Youngster I, Russell GH, Pindar C, Ziv-Baran T, Sauk J, et al. Oral, capsulized, frozen fecal microbiota transplantation for relapsing Clostridium difficile infection. JAMA. 2014; 312: 1772-1778. PubMed: https://pubmed.ncbi.nlm.nih.gov/25322359/

16. Kao D, Roach B, Silva M, Beck P, Rioux K, et al. Effect of Oral Capsule- vs Colonoscopy-Delivered Fecal Microbiota Transplantation on Recurrent Clostridium difficile infection. A Randomized Clinical Trial. JAMA. 2017; 318: 1985-1993.

PubMed: https://pubmed.ncbi.nlm.nih.gov/29183074/
17. Almeida R, Gerbaba T, Petrof EO. Recurrent Clostridium difficile infection and the microbiome. J Gastroenterol. 2016; 51: 1-10. PubMed: https://pubmed.ncbi.nlm.nih.gov/26153514/

18. Goodrich JK, Waters JL, Poole AC, Sutter JL, Koren O, et al. Human genetics shape the gut microbiome. Cell. 2014; 159: 789-799. PubMed: https://pubmed.ncbi.nlm.nih.gov/25417156/

19. Drekonja D, Reich J, Gezahegn S, Greer N, Shaukat A, et al. Fecal Microbiota Transplantation for Clostridium difficile Infection, A Systematic Review. Ann Intern Med. 2015; 162: 630-638. PubMed: https://pubmed.ncbi.nlm.nih.gov/25938992/

20. Rossen NG, MacDonald JK, de Vries EM, D'Haens GR, de Vos WM, Zoetendal EG, Ponsioen CY, Fecal microbiota transplantation as novel therapy in gastroenterology, A systematic review. World $\mathrm{J}$ Gastroenterol. 2015; 21: 5359-5371.

PubMed: https://pubmed.ncbi.nlm.nih.gov/25954111/

21. Moayyedi P, Surette MG, Kim PT, Libertucci J, Wolfe M, et al. Fecal Microbiota Transplantation Induces Remission in Patients with Active Ulcerative Colitis in a Randomized Controlled Trial. Gastroenterology. 2015; 149: 102-109.

PubMed: https://pubmed.ncbi.nlm.nih.gov/25857665/

22. Paramsothy S, Kamm MA, Kaakoush NO, Walsh AJ, van den Bogaerde $\mathrm{J}$, et al. Multidonor intensive faecal microbiota transplantation for active ulcerative colitis, a randomized placebo-controlled trial. Lancet. 2017; 389: 1218-1228. PubMed: https://pubmed.ncbi.nlm.nih.gov/28214091/

23. Rossen NG, Fuentes S, van der Spek MJ, Tijssen JG, Hartman JHA, et al. Findings from a Randomized Controlled Trial of Fecal Transplantation for Patients with Ulcerative Colitis. Gastroenterology. 2015; 149: 110-118.

PubMed: https://pubmed.ncbi.nlm.nih.gov/25836986/

24. Narula N, Kassam Z, Yuan Y, Colombel JF, Ponsioen C, et al. Systematic Review and Meta-analysis, Fecal Microbiota Transplantation for Treatment of Active Ulcerative Colitis. Inflamm Bowel Dis. 2017; 23: 1702-1709.

PubMed: https://pubmed.ncbi.nlm.nih.gov/28906291/

25. Paramsothy S, Kamm MA, Kaakoush NO, Walsh AJ, van den Bogaerde $\mathrm{J}$, et al. Multidonor intensive faecal microbiota transplantation for active ulcerative colitis, a randomized placebocontrolled trial. Lancet. 2017; 389: 1218-1228. PubMed: https://pubmed.ncbi.nlm.nih.gov/28214091/

26. Narula N, Kassam Z, Yuan Y, Colombel JF, Ponsioen C, et al. Systematic Review and Meta-analysis, Fecal Microbiota Transplantation for Treatment of Active Ulcerative Colitis. Inflamm Bowel Dis. 2017; 23: 1702-1709.

PubMed: https://pubmed.ncbi.nlm.nih.gov/28906291/

27. Ishikawa D, Sasaki T, Osada T, Kuwahara-Arai K, Haga K, et al. Changes in Intestinal Microbiota Following Combination Therapy with Fecal Microbial Transplantation and Antibiotics for Ulcerative Colitis. Inflamm Bowel Dis. 2017 23: 116-125.

PubMed: https://pubmed.ncbi.nlm.nih.gov/27893543/

28. Mizuno S, Nanki K, Matsuoka K, Saigusa K, Ono K, et al. Single fecal microbiota transplantation failed to change intestinal microbiota and had limited effectiveness against ulcerative colitis in Japanese patients. Intest Res. 2017; 15: 68-74.

PubMed: https://pubmed.ncbi.nlm.nih.gov/28239315/

29. Nishida A, Imaeda H, Ohno M, Inatomi O, Bamba S, et al. Efficacy and safety of single fecal microbiota transplantation for Japanese patients with mild to moderately active ulcerative colitis. J Gastroenterol. 2017; 52: 476-482.

PubMed: https://pubmed.ncbi.nlm.nih.gov/27730312/

30. Huang $\mathrm{H}, \mathrm{Xu} \mathrm{H}$, Luo $\mathrm{Q}, \mathrm{He} \mathrm{J}$, Li M, et al. Fecal microbiota transplantation to treat Parkinson's disease with constipation, A case report. Medicine. 2019; 98: e16163.

PubMed: https://pubmed.ncbi.nlm.nih.gov/31261545/ 
31. Zhang X, Tian H, Qiao X, Yang B, Hua Y, et al. Efficacy observation of periodic fecal microbiota transplantation in the treatment of refractory constipation.ZhonghuaWeiChang WaiKeZaZhi. 2017;20:1355-1359. PubMed: https://pubmed.ncbi.nlm.nih.gov/29280116/

32. Huang L, Zhu Q, Qu X, Qin H. Microbial treatment in chronic constipation. Science China. Life Sciences. 2018; 61: 744-752. PubMed: https://pubmed.ncbi.nlm.nih.gov/29388040/

33. Ohara $T$, Suzutani $T$. Efficacy of fecal microbiota transplantation in a patient with chronic intractable constipation. Clin Case Rep. 2018; 1-4.

34. Ohara T. Identification of the microbial diversity after fecal microbiota transplantation therapy for chronic intractable constipation using $16 \mathrm{~s}$ rRNA amplicon sequencing. Plos One. 14: e0214085.

PubMed: https://pubmed.ncbi.nlm.nih.gov/30889205/

35. Kootte RS, Levin E, Salojärvi J, Smits LP, Hartstra AV, et al. Improvement of Insulin Sensitivity after Lean Donor Feces in Metabolic Syndrome Is Driven by Baseline Intestinal Microbiota Composition. Cell Metab. 2017; 26: 611-619.

PubMed: https://pubmed.ncbi.nlm.nih.gov/28978426/

36. Vrieze A, van Nood E, Holleman F, Salojärvi J, Kootte RS, et al. Transfer of intestinal microbiota from lean donors increases insulin sensitivity in individuals with metabolic syndrome. Gastroenterology. 2012; 143: 913-916.

PubMed: https://pubmed.ncbi.nlm.nih.gov/22728514/

37. Bajaj JS, Kassam Z, Fagan A, Gavis EA, Liu E, et al. Fecal microbiota transplant from a rational stool donor improves hepatic encephalopathy. A randomized clinical trial. Hepatology. 2017; 66: 1727-1738.

PubMed: https://pubmed.ncbi.nlm.nih.gov/28586116/

38. Bafeta A, Yavchitz A, Riveros C, Batista R, Ravaud P. Methods and Reporting Studies Assessing Fecal Microbiota Transplantation, A Systematic Review. Ann Intern Med. 2017; 167: 34-39. PubMed: https://pubmed.ncbi.nlm.nih.gov/28531908/
39. Cohen NA, Maharshak N. Novel Indications for Fecal Microbial Transplantation, Update and Review of the Literature. Dig Dis Sci. 2017; 62: 1131-1145.

PubMed: https://pubmed.ncbi.nlm.nih.gov/28315032/

40. He Z, Cui BT, Zhang T, Li P, Long CY, et al. Fecal microbiota transplantation cured epilepsy in a case with Crohn's disease, The first report. World J Gastroenterol. 2017; 23: 3565-3568.

PubMed: https://pubmed.ncbi.nlm.nih.gov/28596693/

41. Vujkovic-Cvijin I, Rutishauser RL, Pao M, Hunt PW, Lynch SV, et al Limited engraftment of donor microbiome via one-time fecal microbial transplantation in treated HIV-infected individuals. Gut Microbes. 2017; 8: 440-450.

PubMed: https://pubmed.ncbi.nlm.nih.gov/28541799/

42. The U.S. Food and Drug Administration (FDA). Important Safety Alert Regarding Use of Fecal Microbiota for Transplantation and Risk of Serious Adverse Reactions Due to Transmission of Multi-Drug Resistant Organism. 2019.

43. Cammarota G, laniro G, Gasbarrini A. Faecal microbiota transplantation in clinical practice. Gut. 2017.

44. Ott SJ, Waetzig GH, Rehman A, Moltzau-Anderson J, Bharti R, et al. Efficacy of Sterile Fecal Filtrate Transfer for Treating Patients with Clostridium difficile Infection. Gastroenterology. 2017; 152: 799-811. PubMed: https://pubmed.ncbi.nlm.nih.gov/27866880/

45. Narayanan SP, Bharucha AE. A Practical Guide to Biofeedback Therapy for Pelvic Floor Disorders. Curr Gastroenterol Rep. 2019; 21: 21.

PubMed: https://pubmed.ncbi.nlm.nih.gov/31016468/

46. Nagai Y. Autonomic biofeedback therapy in epilepsy. Epilepsy Res. 2019; 153: 76-78.

PubMed: https://pubmed.ncbi.nIm.nih.gov/30819542/ 